\title{
SOME TAUBERIAN THEOREMS RELATED TO OPERATOR THEORY
}

\author{
C. J. K. BATTY
}

St. John's College, Oxford OX1 3JP, England

This article is a survey of some Tauberian theorems obtained recently in connection with work on asymptotic behaviour of semigroups of operators on Banach spaces. The results in operator theory are described in [6], where we made little attempt to show the Tauberian aspects. At the end of this article, we will give a sketch of the connections between the results in this article and in [6]; for details, the reader can turn to the original papers. In this article, we make no attempt to describe applications of Tauberian theorems in other areas such as number theory and probability theory, apart from a few historical remarks concerning proofs of the Prime Number Theorem.

We begin with a summary of some of the classical Tauberian theorems, which will serve to put the recent results in perspective. Fuller accounts of the classical theory may be found in standard texts such as [9], [26], and in the historical account of van de Lune [24]. In Section 2, we introduce some of the tricks of the trade by applying them to refine the classical theorems. In Section 3, we give the recent results, due to Allan, Arendt, Katznelson, O'Farrell, Prüss, Ransford, Tzafriri and the author [1]-[5], [14], [19], all of which can be obtained from contour integral methods originating in an idea of Newman [17], adapted by Korevaar [15].

Throughout this article, we will state results for the complex-valued case. However, all the results have Banach space-valued versions, and it is those which are required for the applications to operator theory.

1. Some classical theorems. The original theorem of Tauber, which dates from 1897 and from which the subject takes its name, was the following partial converse of Abel's Theorem:

1991 Mathematics Subject Classification: 44-02, 44A10, 40E05, $47 \mathrm{D} 03$.

The paper is in final form and no version of it will be published elsewhere. 
ThEOREM 1.1 [22]. Let $\left(a_{n}\right)_{n \geq 0}$ be a sequence such that $n a_{n} \rightarrow 0$ as $n \rightarrow \infty$ and $\sum_{n=0}^{\infty} a_{n} x^{n} \rightarrow b$ as $x \uparrow 1$. Then $\sum_{n=0}^{\infty} a_{n}=b$.

Theorem 1.1 was extended to the case when $\left(n a_{n}\right)$ is bounded by Hardy [10] and Littlewood [16] in 1910-11.

If we write

$$
\begin{aligned}
s_{n} & =a_{0}+\ldots+a_{n}, \\
h(x) & =\sum_{n=0}^{\infty} a_{n} x^{n}=(1-x) \sum_{n=0}^{\infty} s_{n} x^{n},
\end{aligned}
$$

then we see that, for $0<x<1, h(x)$ is a weighted average of the sequence $\left(s_{n}\right)$. Thus Tauber's Theorem makes an assumption about the asymptotic behaviour of some averages of $\left(s_{n}\right)$, imposes a supplementary condition $\left(n a_{n} \rightarrow 0\right)$, and deduces other information about the asymptotic behaviour of $\left(s_{n}\right)$. Any theorem of this general type is now known as a Tauberian theorem, even when sequences and their associated power series are replaced by functions $f:(0, \infty) \rightarrow \mathbb{C}$ and their Laplace transforms $\widehat{f}$. Indeed, one general principle is that a Tauberian theorem for functions should have two corollaries, one for power series and one for Dirichlet series. For, one may put

$$
f(t)=a_{n} \quad(n \leq t<n+1 ; n=0,1,2, \ldots) .
$$

The Laplace transform of $f$ is

$$
\widehat{f}(z)=\int_{0}^{\infty} e^{-z t} f(t) d t=\left(\frac{1-e^{-z}}{z}\right) h\left(e^{-z}\right),
$$

where $h(x)$ is the power series $\sum_{n=0}^{\infty} a_{n} x^{n}$. Similarly, one may put

$$
f(t)=\sum_{n=1}^{m} \frac{a_{n}}{n} \quad(\log m \leq t<\log (m+1) ; m=1,2, \ldots),
$$

and then

$$
\widehat{f}(z)=\frac{1}{z} \sum_{n=1}^{\infty} \frac{a_{n}}{n^{1+z}} .
$$

We shall draw attention to the versions of some of our results for power series, but we will leave the reader to derive the formulations for Dirichlet series.

The commonest weighted averages of $f$ are its Cesàro means and Abel means. Thus, $f$ is Cesàro convergent to $b$ if $t^{-1} \int_{0}^{t} f(s) d s \rightarrow b$ as $t \rightarrow \infty ; f$ is Abel convergent to $b$ if $\lambda \widehat{f}(\lambda) \rightarrow b$ as $\lambda \downarrow 0$. In general, convergence of $f(t)$ (as $t \rightarrow \infty)$ implies Cesàro convergence, and Cesàro convergence implies Abel convergence [11, 18.2.1]. Any partial converse of these facts is a Tauberian theorem. For example, if $f$ is bounded, then Abel convergence implies Cesàro convergence $[11,18.3 .3]$; if $f$ is non-negative, then Abel convergence implies convergence [26, 8.5.3]. To interpret Tauber's Theorem as a theorem about weighted averages, it was necessary to shift attention from the sequence $\left(a_{n}\right)$ to its partial sums $\left(s_{n}\right)$. Similarly, "Tauberian 
theorems" for functions $f$ may be disguised as results about means of the indefinite integral $F(t):=\int_{0}^{t} f(s) d s$. Since $\lambda \widehat{F}(\lambda)=\widehat{f}(\lambda)$, the following theorem says that Abel convergence of $F$ implies convergence of $F$, under a supplementary condition on (the derivative of) $F$. In the case when $f(t)=a_{n}(n \leq t<n+1)$, it reduces to the extension of Tauber's Theorem obtained by Hardy and Littlewood, and their names are commonly attached to the theorem.

Theorem 1.2 (Hardy-Littlewood) [26, 8.4.3]. Suppose that $f:[0, \infty) \rightarrow \mathbb{C}$ is locally integrable, and that $t f(t)$ is bounded. If $\widehat{f}(\lambda) \rightarrow b$ as $\lambda \downarrow 0$, then $\int_{0}^{t} f(s) d s \rightarrow b$ as $t \rightarrow \infty$.

Although Wiener's Tauberian Theorem is often quoted in a functional analytic form - that certain translation-invariant subspaces, or ideals, of $L^{1}(\mathbb{R})$ are denseit can be formulated in a way which exhibits the Tauberian aspects. If $k \in L^{1}(\mathbb{R})$, $k \geq 0$ and $\int_{-\infty}^{\infty} k(t) d t=1$, then $(k * f)(t)=\int_{-\infty}^{\infty} k(t-s) f(s) d s$ may be thought of as a weighted average of $f$. Thus the following formulation of Wiener's Theorem, published in 1932, assumes convergence of these averages and deduces convergence of other averages of $f$.

TheOREM 1.3 (Wiener) [27], [28, Theorem 4, p. 73], [21, 9.7], [11, 4.21.2]. Let $f \in L^{\infty}(\mathbb{R}), k \in L^{1}(\mathbb{R})$, and suppose that the Fourier transform of $k$ vanishes nowhere in $\mathbb{R}$, and that $(k * f)(t) \rightarrow b \int_{-\infty}^{\infty} k(s) d s$ as $t \rightarrow \infty$. Then $(g * f)(t) \rightarrow$ $b \int_{-\infty}^{\infty} g(s) d s$ as $t \rightarrow \infty$, for any $g$ in $L^{1}(\mathbb{R})$.

Wiener wrote in his autobiography [29, p. 115] that the Tauberian aspects of his work were pointed out to him by Ingham, who spent some time in Göttingen in 1926. Wiener's work, and its developments by Ingham and Ikehara, provided new proofs of the Prime Number Theorem (see, for example, [21, 9.12], [27, p. 233], [28, Chapter III]). Compared with the original methods of Hadamard and de la Vallée Poussin, these proofs avoided the need to estimate the Riemann zeta function at infinity, but they depended on rather difficult results about the Fourier transform.

Apart from writing a monograph [12] on the zeta function, which was based on notes of Littlewood and Bohr, and which became a classic text, Ingham analysed Wiener's work and produced a variety of Tauberian theorems in 1935 [13]. The result which we quote below is a special case of one of them.

If $f \in L^{\infty}(0, \infty)$, then $\widehat{f}(z)$ is defined and holomorphic for $\operatorname{Re} z>0$. We shall be interested in the regular points $i y$ of $\widehat{f}$ in $i \mathbb{R}$, those points in $i \mathbb{R}$ near which $\widehat{f}$ has a holomorphic extension (also denoted by $\widehat{f}$ ). The singular set $i E$ of $\widehat{f}$ in $i \mathbb{R}$ is the set of all points in $i \mathbb{R}$ which are not regular points of $\widehat{f}$.

Theorem 1.4 (Ingham) [13, Theorem I]. Let $f \in L^{\infty}(0, \infty), R>0$, and suppose that all points of $i[-R, R]$ are regular points of $\widehat{f}$. Then

$$
\limsup _{t \rightarrow \infty}\left|\int_{0}^{t} f(s) d s-\widehat{f}(0)\right| \leq \frac{6\|f\|_{\infty}}{R} .
$$


Remark 1.5. The assumption in Theorem 1.4 that $\widehat{f}$ has a holomorphic extension near $i[-R, R]$ can be weakened to assuming that $(\widehat{f}(z)-\widehat{f}(0)) / z$ extends continuously to $i[-R, R]$, for some value of $\widehat{f}(0)$. The results which we give in Section 3 which make assumptions about regular points can also be improved in a similar way, but we will not give the details explicitly.

Ingham's proof of Theorem 1.4 used Fourier analysis, and will not be discussed here. Instead, we shall give in Section 3 a simple proof of Theorem 1.4 due to Korevaar [15].

Although Erdős and Selberg provided an "elementary" proof of the Prime Number Theorem in 1949 (avoiding the use of the zeta function and complex analysis), their argument was lengthy, and the theorem remained rather difficult until 1980 when Newman [17] gave a simple analytical proof, based on a Tauberian theorem for Dirichlet series which could easily be established by estimating a finite contour integral. Korevaar modified Newman's argument to provide a simple proof of Theorem 1.4, and hence of the Prime Number Theorem.

Finally in this section, we want to give one more classical result, due to M. Riesz in 1911 and based on work of Fatou. In Section 2, we shall show how this result can be derived from Theorem 1.4, and we shall obtain an analogous result for the Laplace transform.

Theorem 1.6 (Fatou-Riesz) [20], [23, 7.31]. Let $h(z)$ be holomorphic in the open unit disc with Taylor series $\sum_{n=0}^{\infty} a_{n} z^{n}$. If $a_{n} \rightarrow 0$ as $n \rightarrow \infty$, then $\sum_{n=0}^{\infty} a_{n} \zeta^{n}=h(\zeta)$ for all regular points $\zeta$ of $h$ in the unit circle.

2. Some refinements. In this section, we describe some ways of refining the classical results. The first of these is the notion of slowly oscillating functions, which was used by Pitt to refine Wiener's Theorem.

A function $f:(0, \infty) \rightarrow \mathbb{C}($ or $f: \mathbb{R} \rightarrow \mathbb{C})$ is said to be slowly oscillating $($ at $\infty)$ if

$$
\lim _{\delta \downarrow 0} \limsup _{t \rightarrow \infty} \sup _{t \leq s \leq t+\delta}|f(t)-f(s)|=0
$$

that is, if for all $\varepsilon>0$ there exist $\delta>0$ and $K$ such that

$$
|f(t)-f(s)|<\varepsilon \quad \text { whenever } K \leq t \leq s \leq t+\delta .
$$

Any uniformly continuous function is slowly oscillating, but slowly oscillating functions may be discontinuous. (The reader should be warned that there is not universal agreement about terminology in this area; our terminology follows [11] and $[21]$.

Corollary 2.1 (Pitt) [18], [21, 9.7]. Suppose that, in addition to the assumptions of Theorem 1.3, $f$ is slowly oscillating. Then $f(t) \rightarrow b$ as $t \rightarrow \infty$.

Proof. Let $h=\delta^{-1} \chi_{(0, \delta)}$. By $(2.1),|(h * f)(t)-f(t)|<\varepsilon$ whenever $t \geq K$, so the result follows from Theorem 1.3. 
We can also apply this concept to obtain the following corollary of the HardyLittlewood Theorem.

Corollary $2.2[4$, Theorem 2.10]. Let $f:[0, \infty) \rightarrow \mathbb{C}$ be locally integrable, and suppose that

$$
\limsup _{t \rightarrow \infty} \sup _{t \leq s \leq t+1} t|f(t)-f(s)|<\infty
$$

and that $\lambda \widehat{f}(\lambda) \rightarrow b$ as $\lambda \downarrow 0$. Then $f(t) \rightarrow b$ as $t \rightarrow \infty$.

Proof. For $0<\delta<1$, let

$$
f_{\delta}(t)=\frac{1}{\delta}(f(t+\delta)-f(t)) .
$$

Then

$$
\begin{aligned}
\widehat{f}_{\delta}(\lambda) & =\left(\frac{e^{\delta \lambda}-1}{\delta}\right) \widehat{f}(\lambda)-\frac{1}{\delta} \int_{0}^{\delta} e^{-(t-\delta) \lambda} f(t) d t \\
& \rightarrow b-\frac{1}{\delta} \int_{0}^{\delta} f(t) d t \quad \text { as } \lambda \downarrow 0 .
\end{aligned}
$$

By $(2.2), t f_{\delta}(t)$ is bounded (at least for $t$ sufficiently large, which is adequate for the Hardy-Littlewood Theorem). By Theorem 1.2,

It follows that

$$
\int_{0}^{t} f_{\delta}(s) d s \rightarrow b-\frac{1}{\delta} \int_{0}^{\delta} f(s) d s \quad \text { as } t \rightarrow \infty .
$$

$$
\frac{1}{\delta} \int_{t}^{t+\delta} f(s) d s \rightarrow b \quad \text { as } t \rightarrow \infty .
$$

It follows from (2.2) that $f$ is slowly oscillating, so

$$
\lim _{\delta \downarrow 0} \limsup _{t \rightarrow \infty}\left|\frac{1}{\delta} \int_{t}^{t+\delta} f(s) d s-f(t)\right|=0 .
$$

Hence, $f(t) \rightarrow b$ as $t \rightarrow \infty$.

Remark 2.3. Although Corollary 2.2 is derived from the Hardy-Littlewood Theorem, it is an "improvement" of that theorem in the sense that the theorem can be recovered from the corollary, by applying the corollary to $F(t):=$ $\int_{0}^{t} f(s) d s$.

Now we turn to some consequences of Theorem 1.4. 
Corollary 2.4. Let $f \in L^{\infty}(0, \infty)$, and suppose that one of the following conditions holds:

(1) the singular set of $\widehat{f}$ on $i \mathbb{R}$ is empty,

(2) $f(t) \rightarrow 0$ as $t \rightarrow \infty$.

Then $\int_{0}^{t} e^{-i y s} f(s) d s \rightarrow \widehat{f}(i y)$ as $t \rightarrow \infty$, for all regular points iy of $\widehat{f}$ in $i \mathbb{R}$.

P r o of. First, suppose that $y=0$. In case (1), Corollary 2.4 follows from Theorem 1.4 by letting $R \rightarrow \infty$. In case (2), it follows by replacing $f$ by $f \chi_{(a, \infty)}$ and letting $a \rightarrow \infty$.

For general $y$, the result is derived from the case $y=0$, by replacing $f(t)$ by $e^{-i y t} f(t)$.

Compared with the Hardy-Littlewood Theorem, Corollary 2.4 has replaced the assumption that $t f(t)$ is bounded by the weaker assumption that $f$ is bounded (or $f(t) \rightarrow 0$ ). On the other hand, even for $y=0$, Corollary 2.4 makes assumptions about $\widehat{f}(z)$ for complex $z$ (in the assumptions of regularity), whereas the HardyLittlewood Theorem makes assumptions on $\widehat{f}(\lambda)$ only for real $\lambda$.

Corollary 2.4(2) is a partial analogue of the Fatou-Riesz result (Theorem 1.6) for power series, and we can use it to give a proof of Theorem 1.6 and of another partial analogue for the Laplace transform (Corollary 2.5 below). Corollary 2.4(1) provides no information about power series. If $h$ is holomorphic in an open set containing the closed unit disc, then $h(\zeta)$ is given by the Taylor series whenever $|\zeta|=1$, by elementary complex variable theory.

Proof of Theorem 1.6. Define $f(t)=a_{n}(n \leq t<n+1 ; n=0,1,2, \ldots)$. Then $f(t) \rightarrow 0$ as $t \rightarrow \infty$, and

$$
\widehat{f}(z)=\left(\frac{1-e^{-z}}{z}\right) h\left(e^{-z}\right) \quad(\operatorname{Re} z>0) .
$$

If $\zeta=e^{-i y}(-\pi<y \leq \pi)$ is regular for $h$, then $i y$ is regular for $\widehat{f}$, so

$$
\int_{0}^{N} e^{-i y s} f(s) d s \rightarrow\left(\frac{1-e^{-i y}}{i y}\right) h(\zeta) \quad \text { as } N \rightarrow \infty
$$

by Corollary 2.4 (where $\left(1-e^{-i y}\right) /(i y)$ is to be interpreted as 1 if $\left.y=0\right)$. But

$$
\int_{0}^{N} e^{-i y s} f(s) d s=\frac{1-e^{-i y}}{i y} \sum_{n=0}^{N-1} a_{n} \zeta^{n},
$$

so the result follows.

Corollary 2.5 [5, Corollary 2.5]. Let $f:[0, \infty) \rightarrow \mathbb{C}$ be locally integrable, and suppose that $\widehat{f}(z)$ exists for $\operatorname{Re} z>0$ and that $\lim _{t \rightarrow \infty} \int_{0}^{t} e^{-i \eta s} f(s) d s$ exists for some real $\eta$. Then $\int_{0}^{t} e^{-i y s} f(s) d s \rightarrow \widehat{f}(i y)$ for all regular points iy of $\widehat{f}$ in $i \mathbb{R}$. 
Proof. We may assume that $y \neq \eta$. Replacing $f(t)$ by $e^{-i \eta t} f(t)$, we may assume that $\eta=0$. Define $F(t)=\int_{0}^{t} f(s) d s-c$, where $c=\lim _{t \rightarrow \infty} \int_{0}^{t} f(s) d s$. Then $F(t) \rightarrow 0$ as $t \rightarrow \infty$, and $z \widehat{F}(z)=\widehat{f}(z)-c$. Applying Corollary 2.4(2) to $F$, we find that

$$
i y \int_{0}^{t} e^{-i y s} F(s) d s \rightarrow i y \widehat{F}(i y)=\widehat{f}(i y)-c
$$

But

$$
i y \int_{0}^{t} e^{-i y s} F(s) d s=\int_{0}^{t} e^{-i y s} f(s) d s-e^{-i y t}\left(\int_{0}^{t} f(s) d s-c\right)-c,
$$

so the result follows.

3. The recent results. We begin this section by giving Korevaar's proof of Theorem 1.4. In fact, we will set out the proof in a way which justifies Remark 1.5.

Proof of Theorem 1.4 [15]. Firstly, replacing $f(t)$ by $f(t)-\widehat{f}(0) e^{-t}$, we may assume that $\widehat{f}(0)=0$. Thus the function $\widehat{f}(z) / z$, defined for $\operatorname{Re} z>0$, extends continuously to $i[-R, R]$.

Let $\gamma_{+}$and $\gamma_{-}$be the right and left halves of the circle $|z|=R$, and let $\gamma^{\prime}$ be the line-segment from $i R$ to $-i R$. Let $f_{t}=f \chi_{(0, t)}$, so that $\widehat{f}_{t}$ is holomorphic in the entire complex plane. By Cauchy's Theorem,

$$
\begin{aligned}
\int_{0}^{t} f(s) d s= & \frac{1}{2 \pi i} \int_{\gamma_{+} \cup \gamma_{-}}\left(1+\frac{z^{2}}{R^{2}}\right) \widehat{f_{t}}(z) e^{t z} \frac{d z}{z} \\
& -\frac{1}{2 \pi i} \int_{\gamma_{+} \cup \gamma^{\prime}}\left(1+\frac{z^{2}}{R^{2}}\right) \frac{\widehat{f}(z)}{z} d z \\
= & \frac{1}{2 \pi i} \int_{\gamma_{+}}\left(1+\frac{z^{2}}{R^{2}}\right)\left(\widehat{f_{t}}(z)-\widehat{f}(z)\right) e^{t z} \frac{d z}{z} \\
& -\frac{1}{2 \pi i} \int_{\gamma^{\prime}}\left(1+\frac{z^{2}}{R^{2}}\right) \widehat{f}(z) e^{t z} \frac{d z}{z} \\
& +\frac{1}{2 \pi i} \int_{\gamma_{-}}\left(1+\frac{z^{2}}{R^{2}}\right) \widehat{f_{t}}(z) e^{t z} \frac{d z}{z} .
\end{aligned}
$$

As $t \rightarrow \infty$, the second integral in (3.1) tends to 0 , by the Riemann-Lebesgue Lemma. On $\gamma_{ \pm}$,

$$
\left|1+\frac{z^{2}}{R^{2}}\right|=\frac{2|\operatorname{Re} z|}{R} .
$$


On $\gamma_{+}$,

$$
\left|\left(\widehat{f}_{t}(z)-\widehat{f}(z)\right) e^{t z}\right|=\left|\int_{t}^{\infty} e^{-(s-t) z} f(s) d s\right| \leq \frac{\|f\|_{\infty}}{\operatorname{Re} z}
$$

On $\gamma_{-}$,

$$
\left|\widehat{f}_{t}(z) e^{t z}\right|=\left|\int_{0}^{t} e^{-(s-t) z} f(s) d s\right| \leq \frac{\|f\|_{\infty}}{|\operatorname{Re} z|} .
$$

Thus the first and third integrals in (3.1) are both bounded by $\|f\|_{\infty} / R$, and it follows that

$$
\limsup _{t \rightarrow \infty}\left|\int_{0}^{t} f(s) d s\right| \leq \frac{2\|f\|_{\infty}}{R}
$$

The proof above obtains an estimate for $\limsup _{t \rightarrow \infty}\left|\int_{0}^{t} f(s) d s-\widehat{f}(0)\right|$ which differs from Ingham's estimate by a constant factor. Other estimates were given in [5, Theorem 2.1, Proposition 4.1], using a method based on that of Ransford who obtained analogous estimates for power series [19, Theorems 1.1, 2.1]. Similar estimates may be found in Ingham's paper [13].

EXAMPLE 3.1. Let $f(t)=e^{i a t}$ for some non-zero real number $a$. Then $\widehat{f}(z)=$ $(z-i a)^{-1}$, and $E=\{a\}$. On letting $R \rightarrow a$, the estimate above gives:

$$
\limsup _{t \rightarrow \infty}\left|\int_{0}^{t} f(s) d s-\widehat{f}(0)\right| \leq \frac{2}{|a|} .
$$

In fact, $\int_{0}^{t} f(s) d s=\left(e^{i a t}-1\right) /(i a)$, and

$$
\limsup _{t \rightarrow \infty}\left|\int_{0}^{t} f(s) d s-\widehat{f}(0)\right|=\frac{1}{|a|} .
$$

As observed in Section 2, Corollary 2.4(2) is of no interest for power series. The first non-trivial case for power series is when there is exactly one singular point in the unit circle. This case was resolved by Katznelson and Tzafriri.

THEOREM 3.2 [14]. Let $\left(a_{n}\right)$ be a bounded sequence, let $h(z)=\sum_{n=0}^{\infty} a_{n} z^{n}$, and suppose that $h$ has singular set $\{1\}$ in the unit circle. Then $a_{n}-a_{n+1} \rightarrow 0$ as $n \rightarrow \infty$.

Corollary 3.3. Let $\left(b_{n}\right)$ be a sequence such that $\sup _{N}\left|\sum_{n=0}^{N} b_{n}\right|<\infty$, let $g(z)=\sum_{n=0}^{\infty} b_{n} z^{n}$, and suppose that $g$ has singular set $\{1\}$ in the unit circle. Then $b_{n} \rightarrow 0$ as $n \rightarrow \infty$, and $g(\zeta)=\sum_{n=0}^{\infty} b_{n} \zeta^{n}$, whenever $|\zeta|=1, \zeta \neq 1$.

The proof of Theorem 3.2 in [14] used Fourier analysis. Corollary 3.3 follows by putting $a_{n}=b_{1}+\ldots+b_{n}, h(z)=g(z)(1-z)^{-1}$.

The step from the case of one singular point to null sets of singular points was taken by Allan, O'Farrell, and Ransford [1], by adapting the contour integrals of 
Newman and Korevaar. The argument was carried through in the case of Laplace transforms in [2].

TheOREM 3.4 [2, Theorem 4.1]. Let $f \in L^{\infty}(0, \infty)$, and suppose that the singular set $i E$ of $\widehat{f}$ on $i \mathbb{R}$ is null (for one-dimensional Lebesgue measure). Suppose also that, for all $R>0$,

$$
M(R):=\sup _{\substack{\eta \in E \\|\eta| \leq R}} \sup _{t \geq 0}\left|\int_{0}^{t} f(s) e^{-i \eta s} d s\right|<\infty .
$$

Then $\int_{0}^{t} e^{-i y s} f(s) d s \rightarrow \widehat{f}(i y)$ as $t \rightarrow \infty$, for all regular points iy.

COROLlaRY 3.5 [1]. Suppose that $h(z)=\sum_{n=0}^{\infty} a_{n} z^{n}$ is holomorphic in the open unit disc with singular set $E$ in the unit circle $\Gamma$, suppose that $E$ is null (for Haar measure on the circle), and that

$$
\sup _{\zeta \in E} \sup _{N}\left|\sum_{n=0}^{N} a_{n} \zeta^{n}\right|<\infty .
$$

Then $\sum_{n=0}^{\infty} a_{n} z^{n}=h(z)$ for all regular points $z$ in $\Gamma$.

Corollary 3.5 is deduced from Theorem 3.4 by the standard method of putting $f(t)=a_{n}(n \leq t<n+1 ; n \geq 0)$. Note that the assumptions of the corollary imply that $\left(a_{n}\right)$ is bounded.

In the circumstances of Theorem 3.4 (or Corollary 3.5), convergence may fail at singular points [1, p. 539].

It is not known whether the condition that $E$ is null can be omitted from Theorem 3.4 and Corollary 3.5. In the latter case, it is sufficient to establish that $a_{n} \rightarrow 0$ (Theorem 1.6). When $E=\Gamma$, the other assumptions imply that $h \in H^{\infty}(\Gamma)$, so convergence takes place almost everywhere on $\Gamma$, by Carleson's Theorem [8]. In particular, $a_{n} \rightarrow 0$.

A variant of Theorem 3.4 was given in [2, Theorem 4.4] in which the assumption that $M(R)$ is finite is replaced by the assumption that for each $\eta \in E$, there exists $\delta_{\eta}>0$ such that

$$
\sup \left\{|\widehat{f}(z)|: \operatorname{Re} z>0,|z-i \eta|<\delta_{\eta}\right\}<\infty .
$$

This was proved in [2] by contour integral methods, but it can also be deduced from Ingham's proof of Theorem 1.4 [13].

Theorem 3.4 is derived from the following proposition, in which $\Phi_{k}$ denotes an explicit function of $3 k+2$ real variables, the formula for which may be found in [2]. This proposition plays an important role in the applications to operator theory (see Section 4).

Proposition 3.6 [2, Lemma 3.1]. Let $f \in L^{\infty}(0, \infty)$, let $i E$ be the set of all singular points of $\widehat{f}$ in $i \mathbb{R}$, and suppose that $0 \notin E$. Let $R>0$ and let $I_{j}(j=$ $1, \ldots, k)$ be disjoint open intervals in $(-R, R)$ such that $E \cap[-R, R] \subseteq \bigcup_{j=1}^{k} I_{j}$. 
Let $\varepsilon_{j}$ be half the length of $I_{j}, \xi_{j}$ be the midpoint of $I_{j}, \eta_{j}$ be any point in $I_{j}$, and suppose that

$$
M_{j}:=\sup \left|\int_{0}^{t} e^{-i \eta_{j} s} f(s) d s\right|<\infty .
$$

Then

$$
\limsup _{t \rightarrow \infty}\left|\int_{0}^{t} f(s) d s-\widehat{f}(0)\right| \leq \Phi_{k}\left(\|f\|_{\infty}, R, M_{j}, \varepsilon_{j}, \xi_{j}\right) .
$$

Proposition 3.6 is proved by making the following two modifications to the contour integral in the proof of Theorem 1.4 given at the start of this section:

(a) In the contour $\gamma^{\prime}$, each interval $i I_{j}$ is replaced by a semicircular indentation into the right half-plane;

(b) The integrand is adjusted by including, for each $j=1, \ldots, k$, a suitable factor which vanishes at the two endpoints of $i I_{j}$ (analogous to the factor $1+z^{2} / R^{2}$ which vanishes at the two endpoints of $i[-R, R])$.

Now suppose that $E$ is null. For any $\varepsilon>0$, it is possible to choose $k$ and the intervals $I_{j}$ in such a way that $\varepsilon_{j}=\theta$ for all $j$, where $k \theta<\varepsilon$. Then the estimate in Proposition 3.6 leads to

$$
\limsup _{t \rightarrow \infty}\left|\int_{0}^{t} f(s) d s-\widehat{f}(0)\right| \leq \frac{2 e\|f\|_{\infty}}{R}+\kappa(R) M(R) \varepsilon,
$$

where $\kappa(R)$ depends on $R$ (and on the set $E$ ), but not on $\varepsilon$. Letting $\varepsilon \downarrow 0$ and then $R \rightarrow \infty$ gives Theorem 3.4 .

Similar methods to Section 2 give a corollary of Theorem 3.4 in which the assumption of boundedness is replaced by slow oscillation. This was first carried out in [5], where attention was focussed on Laplace-Stieltjes transforms. The following formulation, due to Arendt and Prüss [4], differs slightly from [5] (see Remark 3.8.5).

Corollary 3.7 [5, Corollary 2.6], [4, Theorem 3.5]. Let $f:[0, \infty) \rightarrow \mathbb{C}$ be locally integrable and slowly oscillating, and let $i E$ be the singular set of $\widehat{f}(z)$ on $i \mathbb{R}$. Suppose that $E$ is null, and that, for all $R>0$,

$$
\sup _{\substack{\eta \in E \\|\eta| \leq R \\ \eta \neq 0}} \sup _{t \geq 0}\left|\int_{0}^{t} e^{-i \eta s} f(s) d s\right|<\infty .
$$

If 0 is either a regular point of $\widehat{f}$, or a pole of order 1 , then $f(t) \rightarrow b$ as $t \rightarrow \infty$, where $b$ is the residue of $\widehat{f}$ at 0 .

Remark 3.8. 1. If $f$ is slowly oscillating, it does not follow that $e^{-i y t} f(t)$ is slowly oscillating. However, if $f$ is (eventually) bounded and slowly oscillating, then $e^{-i y t} f(t)$ is slowly oscillating. 
2. If $f$ is slowly oscillating, $E$ is null, and, for all $R>0$,

$$
\sup _{\substack{\eta \in E \\|\eta| \leq R}} \sup _{t \geq 0}\left|\int_{0}^{t} e^{-i \eta s} f(s) d s\right|<\infty
$$

then $f(t) \rightarrow 0$ as $t \rightarrow \infty$. If $0 \notin E$, this follows from Corollary 3.7. If $0 \in E$, then (3.3) implies that $\sup _{t \geq 0}\left|\int_{0}^{t} f(s) d s\right|<\infty$. It follows directly from this and the slow oscillation of $f$ that $f$ is bounded. For any $y$ in $\mathbb{R} \backslash E, e^{-i y t} f(t)$ is slowly oscillating and Corollary 3.7 implies that $e^{-i y t} f(t) \rightarrow 0$, so $f(t) \rightarrow 0$.

3. Suppose that $f$ is slowly oscillating, the singular set $E$ of $\widehat{f}$ is null, $\widehat{f}$ has a finite set $i E_{1}$ of poles of order 1 in $i \mathbb{R}$ and, for all $R>0$,

$$
\sup _{\substack{\eta \in E \\|\eta| \leq R \\ \eta \notin E_{1}}} \sup _{t \geq 0}\left|\int_{0}^{t} e^{-i \eta s} f(s) d s\right|<\infty .
$$

Then $f(t)-\sum_{y \in E_{1}} b_{y} e^{i y t} \rightarrow 0$ as $t \rightarrow \infty$, where $b_{y}$ is the residue of $\widehat{f}$ at $i y$. This follows from applying Corollary 3.7 to the slowly oscillating function $f(t)-$ $\sum_{y \in E_{1}} b_{y} e^{i y t}$.

4. To apply Corollary 3.7 to a power series $h(z)=\sum_{n=0}^{\infty} a_{n} z^{n}$, we should put $f(t)=\sum_{n=0}^{m} a_{n}(m \leq t<m+1 ; m \geq 0)$. Then $f$ is slowly oscillating only if $a_{n} \rightarrow 0$ as $n \rightarrow \infty$, in which case the Fatou-Riesz Theorem (Theorem 1.6) shows that $\sum_{n=0}^{\infty} a_{n} \zeta^{n}=h(\zeta)$ for all regular points $\zeta$ in $\Gamma$.

5. If $f$ is of locally bounded variation, then Corollary 3.7 may be considered to be a result about the Laplace-Stieltjes transform $\widetilde{f}$ of $f$ :

$$
\widetilde{f}(z)=\int_{0}^{\infty} e^{-z t} d f(s)=z \widehat{f}(z)-f(0) .
$$

In this interpretation, (3.2) is less natural than the variant

$$
\sup _{\substack{\eta \in E \\|\eta| \leq R \\ \eta \neq 0}} \sup _{t \geq 0}\left|\int_{0}^{t} e^{-i \eta s} d f(s)\right|<\infty \text {. }
$$

Since

$$
\int_{0}^{t} e^{-i \eta s} d f(s)=e^{-i \eta t} f(t)+i \eta \int_{0}^{t} e^{-i \eta s} f(s) d s-f(0),
$$

and since we are not assuming that $f$ is bounded, (3.2) and (3.4) are not equivalent in general. The variant of Corollary 3.7 in which (3.2) is replaced by (3.4) was proved in [5, Corollary 2.6]. It then follows that (3.2) and (3.4) are equivalent under our additional assumptions that $f$ is slowly oscillating, $E$ is null, and 0 is either regular or a simple pole. Although this variant is itself a corollary of 
Theorem 3.4, the theorem can easily be recovered from this variant by replacing $f(t)$ by $\int_{0}^{t} f(s) d s$.

So far, all our results have involved boundedness of $f$, either by assumption or by conclusion. We end by giving two results which are applicable to unbounded functions, where the conclusions concern the Cesàro means. Both results are proved by contour integral methods akin to the proof of Theorem 1.4.

Theorem 3.9 [3, Corollary 1.3]. Let $f:[0, \infty) \rightarrow \mathbb{C}$ be locally integrable, and suppose that $f(t)=O(t)$ as $t \rightarrow \infty$, and that every singular point of $\widehat{f}$ in $i \mathbb{R}$ is a pole of order 1 . Then $f(t)$ is Cesàro convergent to the residue of $\widehat{f}(z)$ at $z=0$, as $t \rightarrow \infty$.

Theorem 3.10 [3, Proposition 1.9]. Let $f:[0, \infty) \rightarrow \mathbb{C}$ be locally integrable, and suppose that $f(t)=O(t)$ as $t \rightarrow \infty$, and that there exist $\delta>0, \varepsilon>0$, and $c<\infty$ such that

$$
\left|\widehat{f}\left(r e^{i \theta}\right)\right| \leq \frac{c}{r(\cos \theta)^{1-\varepsilon}} \quad \text { whenever } 0<r<\delta,-\pi / 2<\theta<\pi / 2 .
$$

Then

$$
\limsup _{t \rightarrow \infty}\left|\frac{1}{t} \int_{0}^{t} f(s) d s\right|<\infty .
$$

EXAMPLE 3.11. 1. Let $f(t)=t e^{i a t}$, where $a$ is a non-zero real number. Then $\widehat{f}(z)=(z-i a)^{-2}$, so 0 is regular. Moreover,

$$
\int_{0}^{t} f(s) d s=\frac{t e^{i a t}}{i a}+\frac{e^{i a t}}{a^{2}}-\frac{1}{a^{2}},
$$

so

$$
\limsup _{t \rightarrow \infty}\left|\frac{1}{t} \int_{0}^{t} f(s) d s\right|=\frac{1}{|a|}
$$

2. Let

$$
f(t)=\sum_{n=1}^{\infty}\left\{1+(1+t)\left(i n-\frac{1}{n^{2}}\right)\right\} \frac{e^{i n t-t / n^{2}}}{n^{3}} .
$$

Then $f(t)=O(t)$ as $t \rightarrow \infty$, and the singular set of $\widehat{f}$ on $i \mathbb{R}$ is empty. By Theorem $3.9, f(t)$ is Cesàro convergent to 0 . However, it is shown in [7, Example 6] that

$$
\limsup _{t \rightarrow \infty}\left|\int_{0}^{t} f(s) d s-\widehat{f}(0)\right| \geq \frac{1}{2} .
$$

4. Applications to operator theory. We note here that the results which have direct applications to operator theory are Corollary 2.4, Theorem 3.2, Proposition 3.6, Corollary 3.7, Theorem 3.9 and Theorem 3.10. 
Corollary 2.4(2) shows that if $\{T(t): t \geq 0\}$ is a bounded $C_{0}$-semigroup with generator $A$, and the spectrum of $A$ is contained in $\{z \in \mathbb{C}: \operatorname{Re} z<0\}$, then $\left\|T(t) A^{-1}\right\| \rightarrow 0$ as $t \rightarrow \infty[2],[6$, Remark 3.4(3)].

Theorem 3.2 shows that if $T$ is a power-bounded operator and $\sigma(T) \cap \Gamma=\{1\}$, then $\left\|T^{n}-T^{n+1}\right\| \rightarrow 0$ as $n \rightarrow \infty$ [14, Theorem 1], [6, Corollary 2.2].

Proposition 3.6 is a major step towards the proof in [14] of the central theorem about stability of $C_{0}$-semigroups [14, Theorem 2.4], [6, Theorem 3.1], and also of its extension to Volterra equations [4, Theorem 5.1], [6, Section 4.4].

Corollary 3.7 (in the case when $E$ is empty) produces a stability theorem for individual elements under $C_{0}$-semigroups [7, Theorem 1], [6, Theorem 4.4].

Theorems 3.9 and 3.10 lead to ergodic theorems for certain $C_{0}$-semigroups [3, Theorems 0.2, 2.1], [6, Theorems 4.1, 4.2].

\section{References}

[1] G. R. Allan, A. G. O'Farrell and T. J. Ransford, A Tauberian theorem arising in operator theory, Bull. London Math. Soc. 19 (1987), 537-545.

[2] W. Arendt and C. J. K. Batty, Tauberian theorems and stability of one-parameter semigroups, Trans. Amer. Math. Soc. 306 (1988), 837-852.

[3] - - - A complex Tauberian theorem and mean ergodic semigroups, preprint.

[4] W. Arendt and J. Pr üss, Vector-valued Tauberian theorems and asymptotic behavior of linear Volterra equations, SIAM J. Math. Anal. 23 (1992), 412-448.

[5] C. J. K. Batty, Tauberian theorems for the Laplace-Stieltjes transform, Trans. Amer. Math. Soc. 322 (1990), 783-804.

[6] - Asymptotic behaviour of semigroups of operators, this volume, 35-52.

[7] C. J. K. Batty and Vũ Quôc Phóng, Stability of individual elements under oneparameter semigroups, Trans. Amer. Math. Soc. 322 (1990), 805-818.

[8] L. Carleson, On convergence and growth of partial sums of Fourier series, Acta Math. 116 (1966), 135-157.

[9] G. Doetsch, Handbuch der Laplace-Transformation, I, Birkhäuser, Basel, 1950.

[10] G. H. Hardy, Theorems relating to the summability and convergence of slowly oscillating series, Proc. London Math. Soc. 8 (1910), 301-320.

[11] E. Hille and R. S. Phillips, Functional Analysis and Semi-groups, Amer. Math. Soc., Providence, 1957.

[12] A. E. Ingham, The Distribution of Prime Numbers, Cambridge Univ. Press, Cambridge, 1932 (reprinted 1990).

[13] -, On Wiener's method in Tauberian theorems, Proc. London Math. Soc. 38 (1935), 458-480.

[14] Y. Katznelson and L. Tzafriri, On power bounded operators, J. Funct. Anal. 68 (1986), 313-328.

[15] J. Korevaar, On Newman's quick way to the prime number theorem, Math. Intelligencer 4 (1982), 108-115.

[16] J. E. Littlewood, On the converse of Abel's theorem on power series, Proc. London Math. Soc. 9 (1911), 434-448.

[17] D. J. New man, Simple analytic proof of the prime number theorem, Amer. Math. Monthly 87 (1980), 693-696.

[18] H. R. Pitt, General Tauberian theorems, Proc. London Math. Soc. 44 (1938), 243-288. 
[19] T. J. Ransford, Some quantitative Tauberian theorems for power series, Bull. London Math. Soc. 20 (1988), 37-44.

[20] M. Riesz, Über eine Satz des Herrn Fatou, J. Reine Angew. Math. 140 (1911), 89-99.

[21] W. Rudin, Functional Analysis, McGraw-Hill, New York, 1973.

[22] A. Tauber, Ein Satz aus der Theorie der unendlichen Reihen, Monatsh. Math. Phys. 8 (1897), 273-277.

[23] E. C. Titchmarsh, The Theory of Functions, Oxford Univ. Press, Oxford, 1932.

[24] J.van de Lune, An Introduction to Tauberian Theory: from Tauber to Wiener, Stichting Math. Centrum, Centrum Wisk. Inform., Amsterdam, 1986.

[25] D. V. Widder, The Laplace Transform, Princeton Univ. Press, Princeton, 1941.

[26] - An Introduction to Transform Theory, Academic Press, New York, 1971.

[27] N. Wiener, Tauberian theorems, Ann. of Math. 33 (1932), 1-100.

[28] - The Fourier Integral and Certain of its Applications, Cambridge Univ. Press, Cambridge, 1933 (reprinted 1988).

[29] —, I am a Mathematician, Doubleday, New York, 1956. 